\title{
Online screening for distress, the 6th vital sign, in newly diagnosed oncology outpatients: randomised controlled trial of computerised
} vs personalised triage

\author{
LE Carlson ${ }^{*, 1,2}$, A Waller' ${ }^{2}$, SL Groff', L Zhong ${ }^{2}$ and BD Bultz',2 \\ 'Department of Psychosocial Resources, Tom Baker Cancer Centre, Alberta Health Senvices-Cancer Care Holy Cross Site, 2202 2nd Street SW, Calgary, \\ Alberta, Canada T2S 3CI; '2Department of Oncology, University of Calgary, Calgary, Alberta, Canada
}

BACKGROUND: This randomised controlled trial examined the impact of screening for distress followed by two different triage methods on clinically relevant outcomes over a 12 -month period.

METHODS: Newly diagnosed patients attending a large tertiary cancer centre were randomised to one of the two conditions: (I) screening with computerised triage or (2) screening with personalised triage, both following standardised clinical triage algorithms. Patients completed the Distress Thermometer, Pain and Fatigue Thermometers, the Psychological Screen for Cancer (PSSCAN) Part $C$ and questions on resource utilisation at baseline, 3, 6 and 12 months.

RESULTS: In all, 3 I 33 patients provided baseline data (67\% of new patients); with 1709 (54.5\%) retained at 12 months (I5.4\% deceased). Mixed effects models revealed that both groups experienced significant decreases in distress, anxiety, depression, pain and fatigue over time. People receiving personalised triage and people reporting higher symptom burden were more likely to access services, which was subsequently related to greater decreases in distress, anxiety and depression. Women may benefit more from personalised triage, whereas men may benefit more from a computerised triage model.

CONCLUSION: Screening for distress is a viable intervention that has the potential to decrease symptom burden up to 12 months post diagnosis. The best model of screening may be to incorporate personalised triage for patients indicating high levels of depression and anxiety while providing computerised triage for others.

British Journal of Cancer (2012) 107, 617-625. doi:10.1038/bjc.2012.309 www.bjcancer.com

Published online 24 July 2012

(C) 2012 Cancer Research UK

Keywords: screening for distress; 6th vital sign; triage

People with cancer often experience levels of distress, physical and psychosocial morbidity that are particularly burdensome (Zabora et al, 2001; Carlson et al, 2004; Graves et al, 2007). Distress is a multifactorial category of emotional suffering that 'extends along a continuum, ranging from common normal feelings of vulnerability, sadness and fears, to problems that can become disabling such as depression, anxiety, panic, social isolation and spiritual crisis' (National Comprehensive Cancer Network I, 2002). It arises from difficulties in physical domains such as pain, fatigue, nutritional concerns, as well as common psychosocial and practical concerns (Bultz et al, 2009). Preliminary work by our team documenting the natural course of distress in newly diagnosed patients over 1 year in a large clinical population suggests that for some people with cancer, distress may decrease as a matter of course; for others, distress may be persistent (Carlson et al, 2011). Also of note was that across all patients, fatigue and pain symptoms did not decrease in this naturalistic setting over the full year (Carlson et al, 2011). Other longitudinal studies have also reported that distress levels may be maintained (Akechi et al, 2006; Andreu et al, 2012) or even increase over time in breast, prostate and lung cancer patients (Wang et al, 2006; Couper et al, 2010).

*Correspondence: Dr LE Carlson; E-mail: Icarlso@ucalgary.ca Received 30 March 2012; revised 31 May 2012; accepted 31 May 2012; published online 24 July 2012
National and international organisations have therefore taken steps to recognise distress as the 6th vital sign in cancer care (Rebalance Focus Action Group, 2005; Accreditation Canada, 2008; Bultz and Groff, 2009; Bultz and Johansen, 2011); and there is an increasing focus on how best to assess and manage patient concerns.

Despite recommendations for the adoption of screening and the limitations associated with relying on clinical acumen alone (Jenkins et al, 2001); few cancer programs routinely screen patients for distress (Jacobsen and Ransom, 2007; Pirl et al, 2007; Vodermaier and Linden, 2008). Of those that have adopted screening for distress, few have incorporated strong research or evaluation components. In 2010, we reported the results of a large clinical trial testing the effects of three versions of screening on subsequent distress in newly diagnosed lung and breast cancer patients (Carlson et al, 2010). Over time, patients who received full screening, feedback and personalised triage to appropriate resources benefited the most. This was particularly evident for lung cancer patients. These results lend support to the feasibility and benefits of screening with personalised triage for decreasing distress (Carlson et al, 2010); however, this model was not completely integrated within the clinic and hence was highly resource intensive.

Considering that acceptability and sustainability are recognised as key determinants of successful implementation of new initiatives 
(Bidstrup et al, 2011; Bultz et al, 2011; Mitchell et al, 2011), best-practice approaches for implementing sustainable and effective screening for distress programs in clinical settings require exploration (Fitch, 2011). The current study sought to investigate whether it was possible to accrue the benefits of personalised triage as seen the previous trial, but by using fewer human resources based upon a patient empowerment model. Hence, we decided to compare computerised screening involving patients receiving immediate personalised feedback regarding resources available to address their concerns, against the personalised triage model which was most effective in the previous trial, in order to determine the most efficacious model of screening.

\section{MATERIALS AND METHODS}

\section{Objectives}

1. To examine the impact of screening for distress followed by either computerised or personalised triage on average levels of distress, pain, fatigue, anxiety and depression at 3, 6 and 12 months following baseline in newly diagnosed cancer patients.

2. To examine the impact of screening for distress followed by either computerised or personalised triage on the proportion of patients experiencing clinically elevated levels of each outcome at each follow-up time point.

3. To examine the services accessed by patients, and associations between service utilisation and changes in clinical outcomes.

\section{Participants}

All new ambulatory oncology patients 18 years and over attending a large tertiary cancer centre in Calgary, Alberta, Canada between October 2007 and March 2009 were eligible for the study. If the person was unable to read or speak English, or was physically unable to complete the screening, then the person was counted as 'excused' and the reason for non-participation was recorded.

\section{Treatment conditions}

Consenting patients completed the online screening tool and were randomly assigned to receive one of the two triage interventions: (1) computerised triage or (2) personalised triage. Triage was provided at the baseline assessment only. Random allocations were undertaken using a computer-based randomisation program in an allocation ratio of $1: 1$

Computerised triage condition Participants received a printout that included a summary of their concerns and instructions on how to access appropriate services based on their scores (Supplementary Appendix 1: computerised triage printout example). Once participants completed the screening questions the kiosk immediately printed the report and prompted them to take it. Receptionists also reminded patients to take their report home. A summary report was also attached to the medical chart. Specific services recommended for each patient were based on triage 'streams' including: (1) psychosocial support; (2) resource counselling; (3) pain management; (4) fatigue management and (5) nutrition management. Patients who indicated thoughts of suicide were directly contacted by a staff member (Figure 1).

Personalised triage condition Participants received a brief computer printout summarising the concerns they indicated and informing them they would be contacted by a member of the screening team within 3 days. Participants were encouraged to take the report home and a summary report was attached to the medical record. Participants were then contacted by phone by a member of the screening team within 3 working days, who discussed referral options based on the same criteria utilised in computerised triage (Figure 1). This condition approximates the personalised triage, which was most effective in our previous study (Carlson et al, 2010).

\section{Triage algorithms}

Triage was provided at the baseline assessment only, not during follow-up assessments. The clinical triage algorithms utilised in this study were based on a stepped model of psychosocial care and specific to services available at our centre, in general funnelling patients from less resource-intensive interventions (classes or 1 day seminars) to more personalised, intensive interventions (counselling or psychiatry) for patients with more complicated needs (Cunningham, 1995; Cunningham and Edmonds, 1996). Interventions offered were empirically supported and derived primarily from cognitive-behavioural and humanistic/existential therapy models (for a more detailed description of services offered, see Waller et al, 2011).
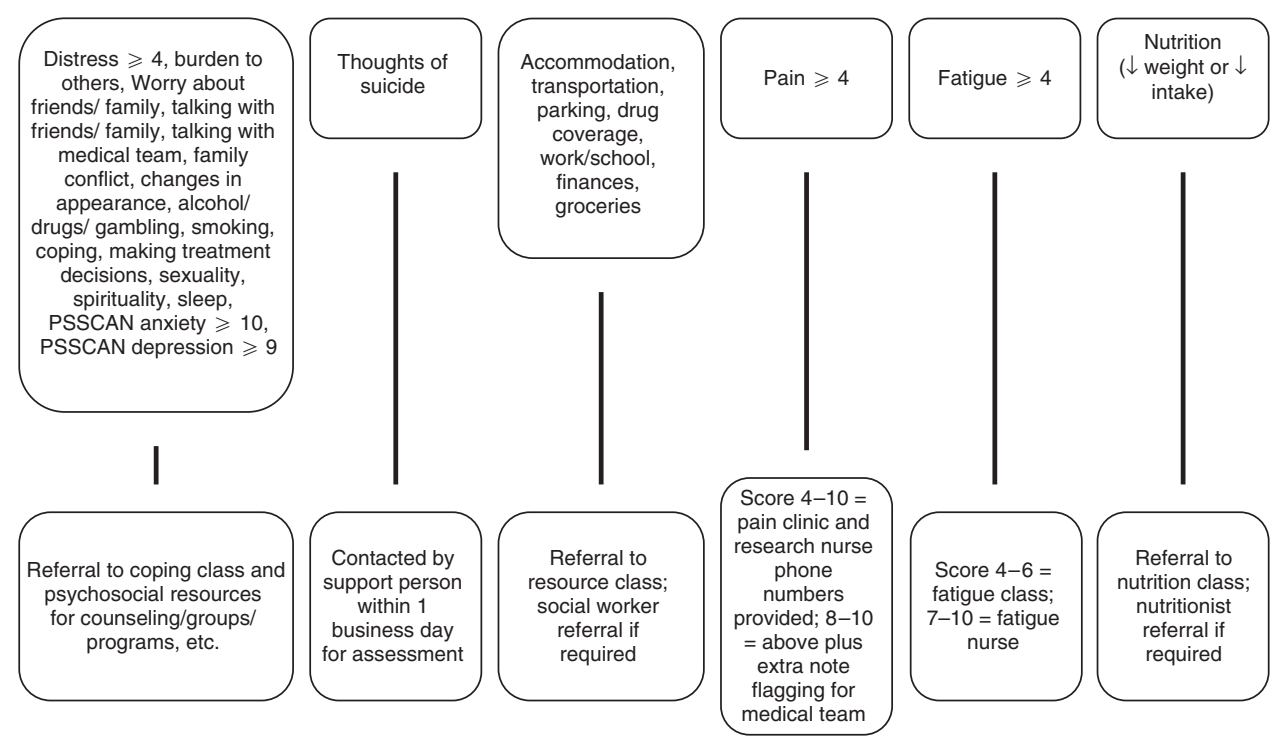

Referral to

nutrition class;

nutritionist

referral if

required

Figure I Screening for distress triage algorithm. 


\section{Screening measures}

1. Demographics and cancer history: Included age, gender, marital status, living arrangements, education, ethnicity, income source and amount, type of cancer and stage of treatment process. Cancer-related variables were confirmed though chart review.

2. The Distress Thermometer (DT): A 0-10 visual analogue scale (VAS) vertically oriented in the form of a usual thermometer. A cutoff score of $\geqslant 4$ performs best in terms of sensitivity and specificity for labelling patients with high distress (Jacobsen et al, 2005; Mitchell, 2007).

3. Pain: A numerical rating scale from 0 to 10 similar to Cleeland and Ryan (Cleeland and Ryan, 1994; Dworkin et al, 2005) was used. A cutoff of $\geqslant 4$ was used to identify cases of pain (Butt et al, 2008).

4. Fatigue: A 0-10 point numeric rating scale similar to the DT. For consistency with the NCCN guidelines (National Comprehensive Cancer Network CRF Panel, 2005), a cutoff of $\geqslant 4$ was used to identify cases of fatigue.

5. The psychological screen for cancer (PSSCAN Part C) (Linden et al, 2005, 2009): Developed for screening in clinical practice and as a research tool, the PSSCAN Part C measures anxiety and depression using 10 items rated on a 5-point Likert scale, ranging from 'not at all' to 'very much so'. A cutoff score of $\geqslant 11$ on each subscale indicates high anxiety and depression (Linden et al, 2005, 2009).

6. Access to services: Participants were asked by the screening assistant at the 3,6 and 12 month follow-up whether they had accessed a range of specific services offered at our centre. A list of the range of services probed with descriptions is available in Waller et al (2011).

\section{Study procedures}

All procedures were approved by the Conjoint Health Research Ethics Board of the University of Calgary, Faculty of Medicine/ TBCC. Screening began in October 2007 and the final 12 month follow-up was completed in March 2010. At their first visit, receptionists directed eligible patients to touch screen kiosks stationed in the waiting areas. Patients were provided with information about the study and completed consent forms and the online screening for distress program at the kiosks. Patients did not have contact with members of the screening team at the time of screening (unless they required assistance or had further questions about the study at the time of consent). This was done in order to implement a model of screening that would be sustainable over time using only existing resources and staffing. The screening team was electronically alerted to the screening results of patients assigned to the personalised group only. The one exception was if a patient indicated thoughts of suicide $(n=213 ; 6.8 \%)$. Regardless of triage group assignment, patients were contacted by a staff member within $24 \mathrm{~h}$ who conducted a suicide assessment and made appropriate referrals. The screening team included people with at least a Psychology undergraduate degree who were specifically trained for this position. Patients completed followup 3, 6 and 12 months post baseline via their choice of phone or e-mail. The computer system was developed specifically for the study over a period of $\sim 6$ months, in partnership with a private IT company. It ran off a dedicated secure server located remotely, and received approval from the health-care system through a privacy impact assessment.

\section{Statistical power}

Based on the data obtained from our previous observational study (Carlson et al, 2011), we used Hierarchical Linear Modelling (HLM) to compare the trend difference of DT between the gender groups and obtain variance estimates. Intercept variance was
2.714, slope variance was 0.004 , covariance between intercept and slope was -0.034 and error variance was 5.327 . We estimated that with 198 participants in each triage group at baseline and assuming the attrition rates were 0.30 ( 3 months), 0.10 ( 6 months) and 0.15 (12 months) for the analysis of distress (Raudenbush and Bryk, 2002), we would have $95 \%$ of power ( $\alpha$ level 0.05 , two-tailed test) to estimate a between group linear trend effect size of 0.5 . As we collected 1531 and 1602 participants at baseline for the computerised and personalised group, respectively, we had $>99 \%$ power ( $\alpha$ level 0.05 , two-tailed test) to estimate a between group linear trend effect size of 0.50 on DT.

\section{Statistical analysis}

The primary end point in this study was distress as measured by the DT over time. The secondary end points were measures of anxiety, depression, pain and fatigue. The HLM was used in the primary analysis, allowing us to treat time as a continuous variable, and handle unbalanced data and unequal spacing conditions caused by variation in testing intervals, in a flexible way (Raudenbush and Bryk, 2002). To assess objective 1, differences between the triage groups at baseline, overall rate of change and rate of change between triage groups on distress, anxiety, depression, pain and fatigue were examined. The approximate linearity was achieved using a logarithmic transformation on anxiety, depression and pain. Cohen's $d$ analogue conveyed effect magnitude. Previous research recommends that for single-item VASs assessing quality of life (or in this case the DT as a proxy measure of a specific domain of quality of life) a 'clinically significant change is suggested as $50 \%$ of the scale's standard deviation'; or a change of 1 on an 11-point numerical scale (Hauser and Walsh, 2008). Others recommend 0.2 s.d. as small change, $0.5 \mathrm{~s} . \mathrm{d}$. as moderate change and $0.8 \mathrm{s.d}$. as large change (Sloan et al, 2006). We also ran two three-way HLM analyses on each of the five outcomes to examine the impact of gender (triage group $\times$ time $\times$ gender) and surgery before baseline (triage group $\times$ time $\times$ surgery).

To assess objective 2 , the $\chi^{2}$ tests compared the percentage of participants in each triage group at risk for clinically significant distress, pain and fatigue using a cutoff score of $\geqslant 4$; and anxiety and depression using a cutoff score of $\geqslant 11$.

To assess objective 3 , a summed score representing the number of people who used services at each time point was created and dichotomised (did not use services/used at least one service). $\chi^{2}$ tests analysed group differences in access at each time point. As outcome cutoff scores were used as the criteria for recommending services to patients (via computerised report or telephone depending on group assignment), we examined whether a higher percentage of people reporting scores above the clinical cutoff for each outcome accessed services, when compared with those who reported scores below the clinical cutoff. Comparisons were made within each triage group at each time point using $\chi^{2}$ tests. Change scores for each outcome were calculated for the intervals between each time point; and independent $t$-tests were conducted on change scores. The HLM models were analysed using Proc Mixed procedure in SAS 9.2 (SAS Institute Inc., Cary, NC, USA, 2007). All remaining data were analysed using SPSS Version 19 (IBM Corp., New York, NY, USA).

\section{RESULTS}

\section{Participants}

Of the 4596 new patients registered during the study, 3133 (67\% of eligible population) provided baseline data and 1709 (54.5\%) were retained at 12 months (Figure 2 - CONSORT diagram for reasons for non-consent and loss to follow-up). A higher percentage of people 


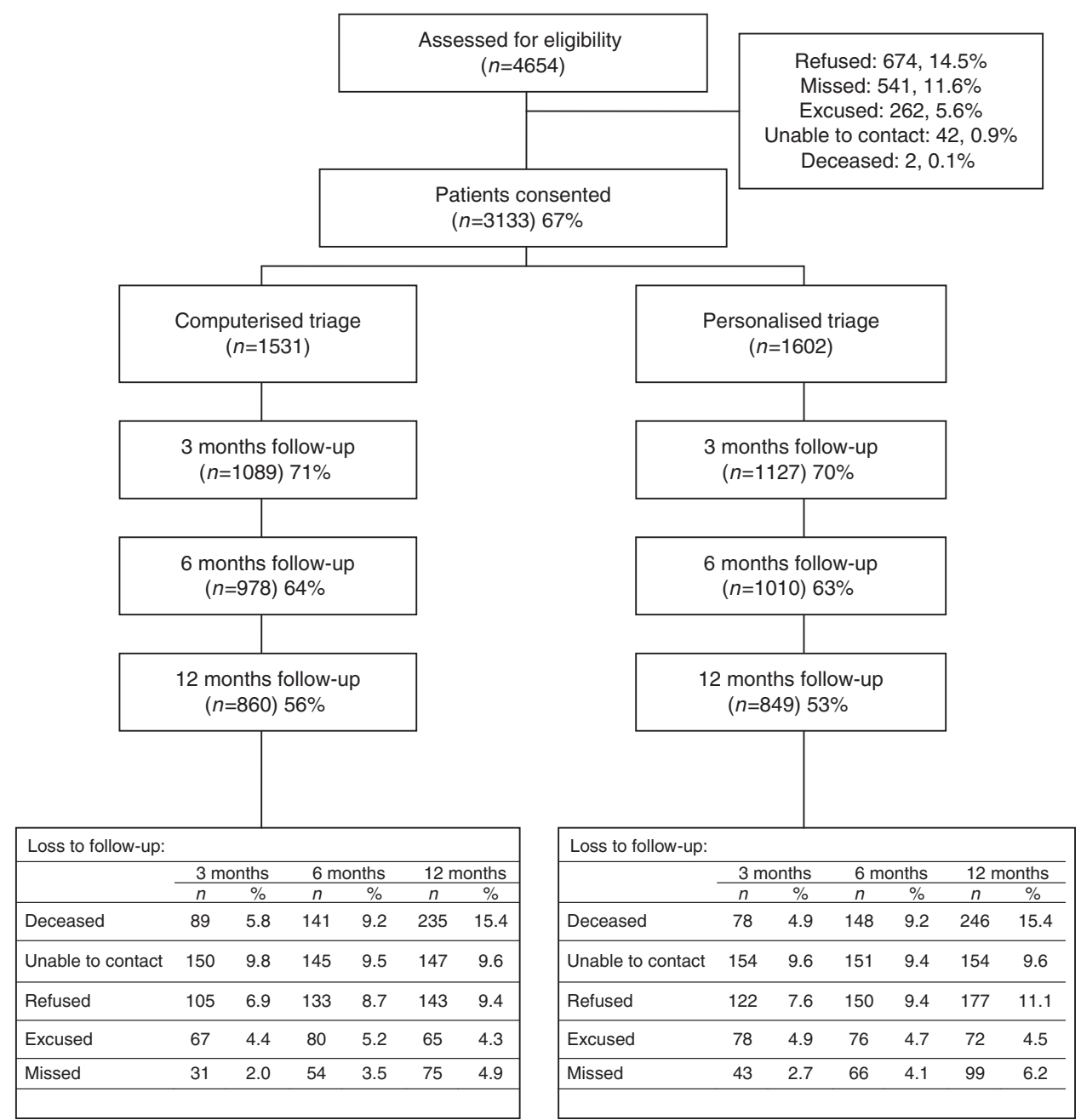

Figure 2 CONSORT recruitment diagram. NB: Excused refers to patients who were too ill to participate, too anxious/upset or did not understand English sufficiently.

in the personalised group had received surgery in the month before baseline; there were no other significant differences between the triage groups on any other baseline variables (Table 1).

\section{Objective 1: changes in outcomes over time}

There were no statistically significant differences between the two triage groups on baseline scores for any outcomes $(P>0.15$ Table 2). There was a significant decline in the rate of change over time for distress, anxiety, depression, pain and fatigue $(P<0.0001)$. We found moderate clinically significant reductions in distress $(0.63$ s.d. for computerised and 0.67 s.d. for personalised) and anxiety ( 0.63 s.d. for computerised and 0.67 s.d. for personalised); a moderate change in pain for the personalised group ( 0.52 s.d.); a smaller reduction in pain for the computerised group ( 0.33 s.d.); and smaller reductions in fatigue $(0.28$ s.d. for computerised and 0.30 s.d. for personalised) and depression ( 0.21 s.d. for computerised and $0.36 \mathrm{s.d}$. for personalised). However, no significant interaction effect was found between rate of change and group $(P>0.15, d<0.1$ for all).

A three-way HLM analysis (triage group $\times$ time $\times$ gender) was conducted to compare females and males on each of the five outcomes across time and between groups (Table 2). A three-way gender $\times$ triage $\times$ time interaction effect was found for the anxiety outcome (Table 2). Males in computerised group improved more than males in personalised group. Females in personalised group improved more than females in computerised group. A two-way interaction (gender $\times$ time) was found for distress and depression. Females improved more than males on both of these outcomes.

A three-way HLM analysis (triage group $\times$ time $\times$ surgery) was conducted to compare patients who had received surgery in the month before baseline to treatment naive patients on each of the five outcomes (Table 2). No three-way interaction effects were found for any outcome. Two-way interactions (surgery $\times$ time) were found for pain, depression, distress and anxiety. People who had received surgery in the month before baseline improved more on these outcomes than treatment naïve patients. The effect sizes for both of these gender and surgery sub-group differences were very small $(d<0.15)$.

\section{Objective 2: prevalence of clinical outcomes across groups}

The computerised triage group had a lower percentage of participants with distress above the clinical cutoff $\left(\chi^{2}=5.27\right.$, $P<0.05)$ compared with the personalised group at 3 months (Figure 3). There were no differences between the triage groups for any other outcomes at any other time points.

\section{Objective 3: service utilisation and changes in clinical outcomes}

During the 12 months, $21.6 \%$ of participants accessed at least one service; these participants accessed an average of 3.69 services $($ s.d. $=3.08)$ (Figure 4). The five most accessed services included 
Table I Participant demographics and medical interventions for participants in computerised and personalised triage groups at baseline

\begin{tabular}{|c|c|c|c|c|}
\hline \multirow[b]{2}{*}{ Demographic and medical interventions } & \multicolumn{2}{|c|}{$\begin{array}{l}\text { Computer } \\
(n=1531)\end{array}$} & \multicolumn{2}{|c|}{$\begin{array}{l}\text { Personalised } \\
(n=1602)\end{array}$} \\
\hline & $\mathbf{N}$ & $\%$ & $\mathbf{N}$ & $\%$ \\
\hline Mean age (years) & 60.83 & & 61.65 & \\
\hline s.d. & 13.55 & & |3.5 | & \\
\hline \multicolumn{5}{|l|}{ Gender } \\
\hline Male & 648 & 42.3 & 665 & 41.5 \\
\hline Female & 883 & 57.7 & 937 & 58.5 \\
\hline \multicolumn{5}{|l|}{ Marital status } \\
\hline Single & 140 & 9.1 & 115 & 7.2 \\
\hline Married & 971 & 63.4 & 1039 & 64.9 \\
\hline Separated & 32 & 2.1 & 41 & 2.6 \\
\hline Divorced & 125 & 8.2 & 122 & 7.6 \\
\hline Widow/widower & 136 & 8.9 & 174 & 10.9 \\
\hline Common law & 85 & 5.6 & 72 & 4.5 \\
\hline Committed & 14 & 0.9 & 10 & 0.6 \\
\hline Missing & 28 & 1.8 & 29 & 1.8 \\
\hline \multicolumn{5}{|l|}{ Living arrangements } \\
\hline Not alone & 1232 & 80.5 & 1282 & 80.0 \\
\hline Alone & 272 & 17.5 & 292 & 18.2 \\
\hline Missing & 27 & 1.8 & 29 & 1.8 \\
\hline \multicolumn{5}{|l|}{ Education } \\
\hline Elementary School (I-6) & 28 & 1.8 & 27 & 1.7 \\
\hline Middle School (7-9) & 139 & 9.1 & 131 & 8.2 \\
\hline High School (10-12) & 526 & 34.4 & 546 & 34.1 \\
\hline Community College & 304 & 19.9 & 304 & 19.0 \\
\hline Some University & 161 & 10.5 & 171 & 10.7 \\
\hline Completed University & 226 & 14.8 & 279 & 17.4 \\
\hline Postgraduate & 119 & 7.8 & 110 & 6.9 \\
\hline Missing & 28 & 1.8 & 34 & 2.1 \\
\hline \multicolumn{5}{|l|}{ Ethnicity } \\
\hline White/Caucasian & 1336 & 87.3 & 1412 & 88.1 \\
\hline Southeast Asian & 42 & 2.7 & 35 & 2.2 \\
\hline South Asian & 21 & 1.4 & 23 & 1.4 \\
\hline First Nation & 15 & 1.0 & 15 & 0.9 \\
\hline Latin American/Hispanic & 15 & 1.0 & 7 & 0.4 \\
\hline Chinese & 33 & 2.2 & 39 & 2.4 \\
\hline Arab/Middle Eastern & 14 & 0.9 & 6 & 0.4 \\
\hline Black & 7 & 0.5 & 18 & 1.1 \\
\hline Multiple ethnicities & 17 & 1.1 & 16 & 1.0 \\
\hline Missing & 31 & 2.0 & 15 & 1.9 \\
\hline \multicolumn{5}{|l|}{ English as first language } \\
\hline Yes & 1335 & 87.2 & 1404 & 87 \\
\hline No & 170 & 11.1 & 169 & 10.7 \\
\hline Missing & 26 & 1.7 & 29 & 1.8 \\
\hline \multicolumn{5}{|l|}{ Family income } \\
\hline Less than $\$ 30000$ & 338 & 22.1 & 340 & 21.2 \\
\hline Less than $\$ 50000$ & 290 & 18.9 & 294 & 18.4 \\
\hline Less than $\$ 80000$ & 220 & 14.4 & 255 & 15.9 \\
\hline Less than $\$ 100000$ & 126 & 8.2 & 127 & 7.9 \\
\hline More than $\$ 100000$ & 249 & 16.3 & 257 & 16.0 \\
\hline Prefer not to say & 280 & 18.3 & 297 & 18.5 \\
\hline Missing & 28 & 1.8 & 32 & 2.0 \\
\hline \multicolumn{5}{|l|}{ Source income } \\
\hline Employment & 490 & 32.0 & 526 & 32.8 \\
\hline Pension/retirement (CPP) & 519 & 33.9 & 537 & 33.5 \\
\hline Family members (spouse/parent) & 171 & 11.0 & 163 & 10.2 \\
\hline Social assistance & 129 & 8.6 & 123 & 7.7 \\
\hline Prefer not to say & 98 & 6.4 & 113 & 7.1 \\
\hline Other & 97 & 6.3 & 108 & 6.7 \\
\hline Missing & 27 & 1.8 & 32 & 2.0 \\
\hline
\end{tabular}

Table I (Continued)

\begin{tabular}{|c|c|c|c|c|}
\hline \multirow[b]{2}{*}{ Demographic and medical interventions } & \multicolumn{2}{|c|}{$\begin{array}{l}\text { Computer } \\
(n=1531)\end{array}$} & \multicolumn{2}{|c|}{$\begin{array}{l}\text { Personalised } \\
\qquad(n=1602)\end{array}$} \\
\hline & $N$ & $\%$ & $\mathbf{N}$ & $\%$ \\
\hline \multicolumn{5}{|l|}{ Diagnosis } \\
\hline Breast & 458 & 29.9 & 489 & 30.5 \\
\hline Gastrointestinal & 256 & 16.7 & 267 & 16.7 \\
\hline Lung & 188 & 12.3 & 202 & 12.6 \\
\hline Prostate & 194 & 12.7 & 182 & 11.4 \\
\hline Skin & 88 & 5.7 & 98 & 6.1 \\
\hline Gynaecologic & 95 & 6.2 & 84 & 5.2 \\
\hline Head and neck & 65 & 4.2 & 67 & 4.2 \\
\hline Lymphoma & 41 & 2.7 & 59 & 3.7 \\
\hline Leukaemia & 29 & 1.9 & 44 & 2.7 \\
\hline Brain & 23 & 1.5 & 27 & 1.7 \\
\hline Thyroid & 23 & 1.5 & 22 & 1.4 \\
\hline Testicular & 20 & 1.3 & 19 & 1.2 \\
\hline Other & 51 & 3.3 & 42 & 2.6 \\
\hline \multicolumn{5}{|l|}{ Treatment before baseline } \\
\hline Surgery & 547 & 35.7 & 627 & 39.1 \\
\hline Chemotherapy & 107 & 7.0 & 88 & 5.5 \\
\hline Radiation therapy & 34 & 2.2 & 41 & 2.6 \\
\hline Hormone therapy & 48 & 3.1 & 42 & 2.6 \\
\hline No treatment & 849 & 55.5 & 857 & 54.7 \\
\hline
\end{tabular}

Abbreviation: $\mathrm{CPP}=$ Canadian Pension Plan

individual counselling, nutritionist, resource social worker, resource class and breast cancer nutrition class (Supplementary Appendix 2). A significantly higher proportion of people in the personalised group accessed services at 3 months $\left(17 \%\right.$ vs $\left.12 \% ; \chi^{2}=9.20, P<0.01\right)$, 6 months $\left(19 \%\right.$ vs $\left.15 \% ; \chi^{2}=7.88, P<0.01\right)$ and 12 months $(20 \%$ vs $15 \%$; $\left.\chi^{2}=9.20, P<0.05\right)$; and over the whole duration of the study $\left(24 \%\right.$ vs $\left.19 \% ; \chi^{2}=9.61, P<0.01\right)$. Overall, 309 patients in the personalised group accessed a total of 1213 services; 236 patients in the computerised group accessed a total of 825 services.

Patients reporting high compared with low distress at baseline in both triage groups were more likely to access services between baseline and the 3-month follow-up (Table 3). Highly depressed patients in both groups were more likely to access services than those with lower depression. Highly anxious and highly fatigued patients were more likely to access services than those with lower levels in the personalised group only. There was a trend for highly anxious patients to access services more than those low on anxiety in the computerised group.

Patients in both triage groups reporting high distress, anxiety and depression at 3 months were more likely to access services between the 3 and 6 month follow-up compared with those reporting lower distress, anxiety and depression (Table 3 ). Highly depressed patients at 6 months were more likely to access services between the 6 and 12 month follow-up in the personalised group only.

Patients who accessed services between the 3 and 6 month follow-up reported a greater decrease in mean distress between 3 and 6 months than patients who did not access services $(t=2.29$, $P<0.05)$. There was a trend for patients who accessed services during this time to report greater decrease in mean anxiety $(t=1.82, P=0.07)$. Patients who accessed services between the 6 and 12 month follow-up reported a greater decrease in mean depression between these time points than patients who did not access services $(t=2.21, P<0.05)$.

\section{DISCUSSION}

Our first objective was to examine any differences between the two triage groups in changes in distress, anxiety, depression, pain and fatigue over time. Patients in both triage groups experienced 
Table 2 Two-way and Three-way HLM significance tests for personalised triage vs computerised triage groups, gender and surgery on intercept and slope estimates $(n=3 \mid 33)$

\begin{tabular}{|c|c|c|c|c|c|}
\hline Outcomes & Parameters & Estimates & s.e. & $t$ & $P$-value \\
\hline \multirow[t]{3}{*}{ Distress } & $\begin{array}{l}\text { Intercept } \\
\text { Time } \\
\text { Triage } \\
\text { Time } \times \text { Triage }\end{array}$ & $\begin{array}{r}3.88 \\
-0.14 \\
0.11 \\
-0.016\end{array}$ & $\begin{array}{l}0.045 \\
0.006 \\
0.090 \\
0.012\end{array}$ & $\begin{array}{r}86.41 \\
-24.54 \\
1.22 \\
-1.37\end{array}$ & $\begin{array}{c}<0.0001 \\
<\mathbf{0 . 0 0 0 1} \\
0.22 \\
0.17\end{array}$ \\
\hline & $\begin{array}{l}\text { Intercept } \\
\text { Gender } \\
\text { Triage } \\
\text { Gender } \times \text { Triage } \\
\text { Time } \\
\text { Time } \times \text { Gender } \\
\text { Time } \times \text { Triage } \\
\text { Time } \times \text { Gender } \times \text { Triage }\end{array}$ & $\begin{array}{r}3.81 \\
-0.78 \\
0.09 \\
-0.22 \\
-0.14 \\
0.02 \\
-0.01 \\
0.009\end{array}$ & $\begin{array}{l}0.04 \\
0.09 \\
0.09 \\
0.18 \\
0.006 \\
0.01 \\
0.01 \\
0.02\end{array}$ & $\begin{array}{r}84.75 \\
-8.64 \\
0.95 \\
-1.20 \\
-23.77 \\
1.96 \\
-1.25 \\
0.40\end{array}$ & $\begin{array}{c}<0.000 \text { I } \\
<\mathbf{0 . 0 0 0 1} \\
0.34 \\
0.23 \\
<\mathbf{0 . 0 0 0 1} \\
\mathbf{0 . 0 5} \\
0.21 \\
0.69\end{array}$ \\
\hline & $\begin{array}{l}\text { Intercept } \\
\text { Surgery } \\
\text { Triage } \\
\text { Surgery } \times \text { Triage } \\
\text { Time } \\
\text { Time } \times \text { Surgery } \\
\text { Time } \times \text { Triage } \\
\text { Time } \times \text { Surgery } \times \text { Triage }\end{array}$ & $\begin{array}{l}3.90 \\
-0.15 \\
0.10 \\
-0.0004 \\
-0.14 \\
0.03 \\
-0.02 \\
0.01\end{array}$ & $\begin{array}{l}0.05 \\
0.09 \\
0.09 \\
0.19 \\
0.006 \\
0.01 \\
0.01 \\
0.02\end{array}$ & $\begin{array}{r}84.22 \\
-1.65 \\
1.13 \\
-0.0 \\
-24.55 \\
2.24 \\
-1.37 \\
0.44\end{array}$ & $\begin{array}{c}<0.0001 \\
0.10 \\
0.26 \\
0.99 \\
<\mathbf{0 . 0 0 0 1} \\
\mathbf{0 . 0 3} \\
0.17 \\
0.66\end{array}$ \\
\hline \multirow[t]{3}{*}{ Fatigue } & $\begin{array}{l}\text { Intercept } \\
\text { Time } \\
\text { Triage group } \\
\text { Time } \times \text { triage group }\end{array}$ & $\begin{array}{r}3.93 \\
-0.06 \\
0.06 \\
-0.02\end{array}$ & $\begin{array}{l}0.05 \\
0.005 \\
0.10 \\
0.01\end{array}$ & $\begin{array}{r}79.73 \\
-10.16 \\
0.61 \\
-1.39\end{array}$ & $\begin{aligned} &< 0.0001 \\
&<\mathbf{0 . 0 0 0 1} \\
& 0.54 \\
& 0.16\end{aligned}$ \\
\hline & $\begin{array}{l}\text { Intercept } \\
\text { Gender } \\
\text { Triage } \\
\text { Gender } \times \text { Triage } \\
\text { Time } \\
\text { Time } \times \text { Gender } \\
\text { Time } \times \text { Triage } \\
\text { Time } \times \text { Gender } \times \text { Triage }\end{array}$ & $\begin{array}{r}3.88 \\
-0.63 \\
0.07 \\
0.16 \\
-0.06 \\
0.02 \\
-0.02 \\
-0.02\end{array}$ & $\begin{array}{l}0.05 \\
0.10 \\
0.10 \\
0.20 \\
0.006 \\
0.01 \\
0.01 \\
0.02\end{array}$ & $\begin{array}{r}78.05 \\
-6.34 \\
0.70 \\
0.82 \\
-9.69 \\
1.76 \\
-1.48 \\
-0.72\end{array}$ & $\begin{array}{c}<0.000 \text { I } \\
<\mathbf{0 . 0 0 0 1} \\
0.49 \\
0.41 \\
<\mathbf{0 . 0 0 0 1} \\
0.08 \\
0.14 \\
0.47\end{array}$ \\
\hline & $\begin{array}{l}\text { Intercept } \\
\text { Surgery } \\
\text { Triage } \\
\text { Surgery } \times \text { Triage } \\
\text { Time } \\
\text { Time } \times \text { Surgery } \\
\text { Time } \times \text { Triage } \\
\text { Time } \times \text { Surgery } \times \text { Triage }\end{array}$ & $\begin{array}{r}3.92 \\
0.08 \\
0.07 \\
-0.07 \\
-0.06 \\
0.02 \\
-0.01 \\
-0.02\end{array}$ & $\begin{array}{l}0.05 \\
0.10 \\
0.10 \\
0.20 \\
0.006 \\
0.01 \\
0.01 \\
0.02\end{array}$ & $\begin{array}{r}77.04 \\
0.74 \\
0.71 \\
-0.34 \\
-10.22 \\
1.36 \\
-1.18 \\
-0.99\end{array}$ & $\begin{array}{c}<0.0001 \\
0.46 \\
0.48 \\
0.73 \\
<\mathbf{0 . 0 0 0 2} \\
0.17 \\
0.24 \\
0.32\end{array}$ \\
\hline \multirow[t]{3}{*}{ Pain (log) } & $\begin{array}{l}\text { Intercept } \\
\text { Time } \\
\text { Triage } \\
\text { Time } \times \text { Triage }\end{array}$ & $\begin{array}{r}0.91 \\
-0.02 \\
-0.04 \\
0.004\end{array}$ & $\begin{array}{l}0.014 \\
0.002 \\
0.03 \\
0.003\end{array}$ & $\begin{array}{r}65.81 \\
-12.34 \\
-1.32 \\
1.33\end{array}$ & $\begin{array}{c}<0.0001 \\
<0.0001 \\
0.19 \\
0.18\end{array}$ \\
\hline & $\begin{array}{l}\text { Intercept } \\
\text { Gender } \\
\text { Triage } \\
\text { Gender } \times \text { Triage } \\
\text { Time } \\
\text { Time } \times \text { Gender } \\
\text { Time } \times \text { Triage } \\
\text { Time } \times \text { Gender } \times \text { Triage }\end{array}$ & $\begin{aligned} & 0.90 \\
- & 0.08 \\
- & 0.04 \\
- & 0.03 \\
- & 0.02 \\
- & 0.0002 \\
& 0.004 \\
& 0.0007\end{aligned}$ & $\begin{array}{l}0.01 \\
0.03 \\
0.03 \\
0.06 \\
0.002 \\
0.003 \\
0.003 \\
0.007\end{array}$ & $\begin{array}{r}64.45 \\
-2.82 \\
-1.42 \\
-0.57 \\
-12.14 \\
-0.04 \\
1.32 \\
0.11\end{array}$ & $\begin{array}{c}<0.0001 \\
\mathbf{0 . 0 0 5} \\
0.16 \\
0.57 \\
<\mathbf{0 . 0 0 0 1} \\
0.96 \\
0.19 \\
0.92\end{array}$ \\
\hline & $\begin{array}{l}\text { Intercept } \\
\text { Surgery } \\
\text { Triage } \\
\text { Surgery } \times \text { Triage } \\
\text { Time } \\
\text { Time } \times \text { Surgery } \\
\text { Time } \times \text { Triage } \\
\text { Time } \times \text { Surgery } \times \text { Triage }\end{array}$ & $\begin{array}{r}0.91 \\
0.02 \\
-0.04 \\
0.01 \\
-0.02 \\
0.01 \\
0.004 \\
0.005\end{array}$ & $\begin{array}{l}0.01 \\
0.03 \\
0.03 \\
0.06 \\
0.002 \\
0.003 \\
0.003 \\
0.007\end{array}$ & $\begin{array}{r}63.56 \\
0.85 \\
-1.29 \\
0.15 \\
-12.51 \\
2.46 \\
1.24 \\
0.72\end{array}$ & $\begin{array}{c}<0.0001 \\
0.39 \\
0.20 \\
0.88 \\
<\mathbf{0 . 0 0 0 1} \\
\mathbf{0 . 0 1} \\
0.21 \\
0.47\end{array}$ \\
\hline & Intercept & 2.06 & 0.006 & 318.58 & $<0.000$ I \\
\hline & $\begin{array}{l}\text { Time } \\
\text { Triage } \\
\text { Time } \times \text { Triage }\end{array}$ & $\begin{array}{r}-0.025 \\
0.001 \\
-0.001\end{array}$ & $\begin{array}{l}0.001 \\
0.013 \\
0.001\end{array}$ & $\begin{array}{r}-35.22 \\
0.09 \\
-1.05\end{array}$ & $\begin{array}{c}<\mathbf{0 . 0 0 0 1} \\
0.93 \\
0.29\end{array}$ \\
\hline & $\begin{array}{l}\text { Intercept } \\
\text { Gender } \\
\text { Triage } \\
\text { Gender } \times \text { Triage } \\
\text { Time } \\
\text { Time } \times \text { Gender } \\
\text { Time } \times \text { Triage } \\
\text { Time } \times \text { Gender } \times \text { Triage }\end{array}$ & $\begin{aligned} & 2.05 \\
&- 0.10 \\
&- 0.004 \\
&- 0.06 \\
&- 0.02 \\
& 0.006 \\
&-0.001 \\
& 0.008\end{aligned}$ & $\begin{array}{l}0.006 \\
0.01 \\
0.01 \\
0.03 \\
0.001 \\
0.001 \\
0.001 \\
0.003\end{array}$ & $\begin{array}{r}316.32 \\
-8.02 \\
-0.35 \\
-2.29 \\
-32.92 \\
3.91 \\
-0.50 \\
2.75\end{array}$ & $\begin{array}{c}<0.0001 \\
<\mathbf{0 . 0 0 0 1} \\
0.73 \\
\mathbf{0 . 0 2} \\
<\mathbf{0 . 0 0 0 1} \\
<\mathbf{0 . 0 0 0 1} \\
0.62 \\
\mathbf{0 . 0 0 6}\end{array}$ \\
\hline
\end{tabular}

Table 2 (Continued)

\begin{tabular}{|c|c|c|c|c|c|}
\hline Outcomes & Parameters & Estimates & s.e. & $t$ & $P$-value \\
\hline & $\begin{array}{l}\text { Intercept } \\
\text { Surgery } \\
\text { Triage } \\
\text { Surgery } \times \text { Triage } \\
\text { Time } \\
\text { Time } \times \text { Surgery } \\
\text { Time } \times \text { Triage } \\
\text { Time } \times \text { Surgery } \times \text { Triage }\end{array}$ & $\begin{aligned} & 2.06 \\
&- 0.03 \\
& 0.003 \\
&- 0.02 \\
&- 0.02 \\
& 0.004 \\
&-0.002 \\
& 0.003\end{aligned}$ & $\begin{array}{l}0.007 \\
0.01 \\
0.01 \\
0.03 \\
0.007 \\
0.001 \\
0.001 \\
0.003\end{array}$ & $\begin{array}{r}309.70 \\
-2.52 \\
0.19 \\
-0.79 \\
-34.26 \\
2.46 \\
-1.17 \\
1.03\end{array}$ & $\begin{array}{c}<0.0001 \\
\mathbf{0 . 0 1} \\
0.85 \\
0.43 \\
<\mathbf{0 . 0 0 0 1} \\
\mathbf{0 . 0 1} \\
0.24 \\
0.30\end{array}$ \\
\hline \multirow{4}{*}{$\begin{array}{l}\text { Depression } \\
\text { (log) }\end{array}$} & Intercept & 1.80 & 0.007 & 272.81 & $<0.0001$ \\
\hline & $\begin{array}{l}\text { Time } \\
\text { Triage group } \\
\text { Time } \times \text { triage group }\end{array}$ & $\begin{array}{r}-0.011 \\
0.006 \\
-0.001\end{array}$ & $\begin{array}{l}0.001 \\
0.013 \\
0.001\end{array}$ & $\begin{array}{r}-16.57 \\
0.48 \\
-0.65\end{array}$ & $\begin{array}{l}<0.0001 \\
0.63 \\
0.51\end{array}$ \\
\hline & $\begin{array}{l}\text { Intercept } \\
\text { Surgery } \\
\text { Triage } \\
\text { Surgery } \times \text { Triage } \\
\text { Time } \\
\text { Time } \times \text { Gender } \\
\text { Time } \times \text { Triage } \\
\text { Time } \times \text { Gender } \times \text { Triage }\end{array}$ & $\begin{aligned} & 1.81 \\
&- 0.06 \\
& 0.007 \\
&-0.02 \\
&-0.01 \\
& 0.02 \\
&-0.01 \\
& 0.009\end{aligned}$ & $\begin{array}{l}0.007 \\
0.01 \\
0.01 \\
0.03 \\
0.001 \\
0.01 \\
0.01 \\
0.02\end{array}$ & $\begin{array}{r}266.06 \\
-4.23 \\
0.50 \\
-0.73 \\
-16.92 \\
1.96 \\
-1.25 \\
0.40\end{array}$ & $\begin{array}{c}<0.0001 \\
<\mathbf{0 . 0 0 0 1} \\
0.62 \\
0.46 \\
<\mathbf{0 . 0 0 0 1} \\
\mathbf{0 . 0 5} \\
0.21 \\
0.69\end{array}$ \\
\hline & $\begin{array}{l}\text { Intercept } \\
\text { Surgery } \\
\text { Triage } \\
\text { Surgery } \times \text { Triage } \\
\text { Time } \\
\text { Time } \times \text { Surgery } \\
\text { Time } \times \text { Triage } \\
\text { Time } \times \text { Surgery } \times \text { Triage }\end{array}$ & $\begin{aligned} & 1.81 \\
&- 0.06 \\
& 0.007 \\
&- 0.02 \\
&- 0.01 \\
& 0.004 \\
&-0.001 \\
& 0.001\end{aligned}$ & $\begin{array}{l}0.007 \\
0.01 \\
0.01 \\
0.03 \\
0.001 \\
0.001 \\
0.001 \\
0.003\end{array}$ & $\begin{array}{r}266.06 \\
-4.23 \\
0.50 \\
-0.73 \\
-16.92 \\
3.06 \\
-0.65 \\
0.47\end{array}$ & $\begin{array}{c}<0.0001 \\
<\mathbf{0 . 0 0 0 1} \\
0.62 \\
0.46 \\
<\mathbf{0 . 0 0 0 1} \\
\mathbf{0 . 0 0 2} \\
0.51 \\
0.64\end{array}$ \\
\hline
\end{tabular}

Abbreviation: HLM $=$ Hierarchical Linear Modelling. Bold entries show significance (using $P<0.05$ as upper limit).

significant decreases in each of the five outcomes. There were no differences between the triage groups on outcomes over time. This could be interpreted either as indicating neither group was effective, or that both were equally effective.

To put this finding in context, an earlier observational study at the same centre (Carlson et al, 2011) found that while distress, depression and anxiety decreased over time; pain and fatigue persisted. In the current intervention study, pain and fatigue as well as distress, depression and anxiety decreased significantly in both groups. This evidence supports the efficacy of both triage methods above usual care in helping to decrease fatigue and pain levels over the year following diagnosis. Clinically, this is an important finding as pain and fatigue are two commonly reported symptoms both in our previous work and that of others, often disturbing quality of life and the ability to function on a daily basis (Carlson et al, 2004; Morrow et al, 2005; Holland et al, 2007). These results are equivocal, however, in terms of the benefits of triage for decreasing distress, anxiety and depression compared with usual care.

Small differences were found when outcomes were examined by gender and receipt of surgery. Overall, females' distress and depression levels improved more than males over the 12 months. Males in the computerised group improved more than males in the personalised group, while females in the personalised group improved more than females in the computerised group. While the effects sizes were small, these findings suggest that women may find particular benefit from screening followed by triage, whereas a model of self-referral on the advice of computerised triage may be more suited to men.

The real benefits of personalised triage above computerised triage alone appeared in further analysis of the use of services by participants in each group. Patients who accessed services experienced greater decreases in distress, anxiety and depression at subsequent screening time points regardless of group assignment, and patients who received personalised triage were more 


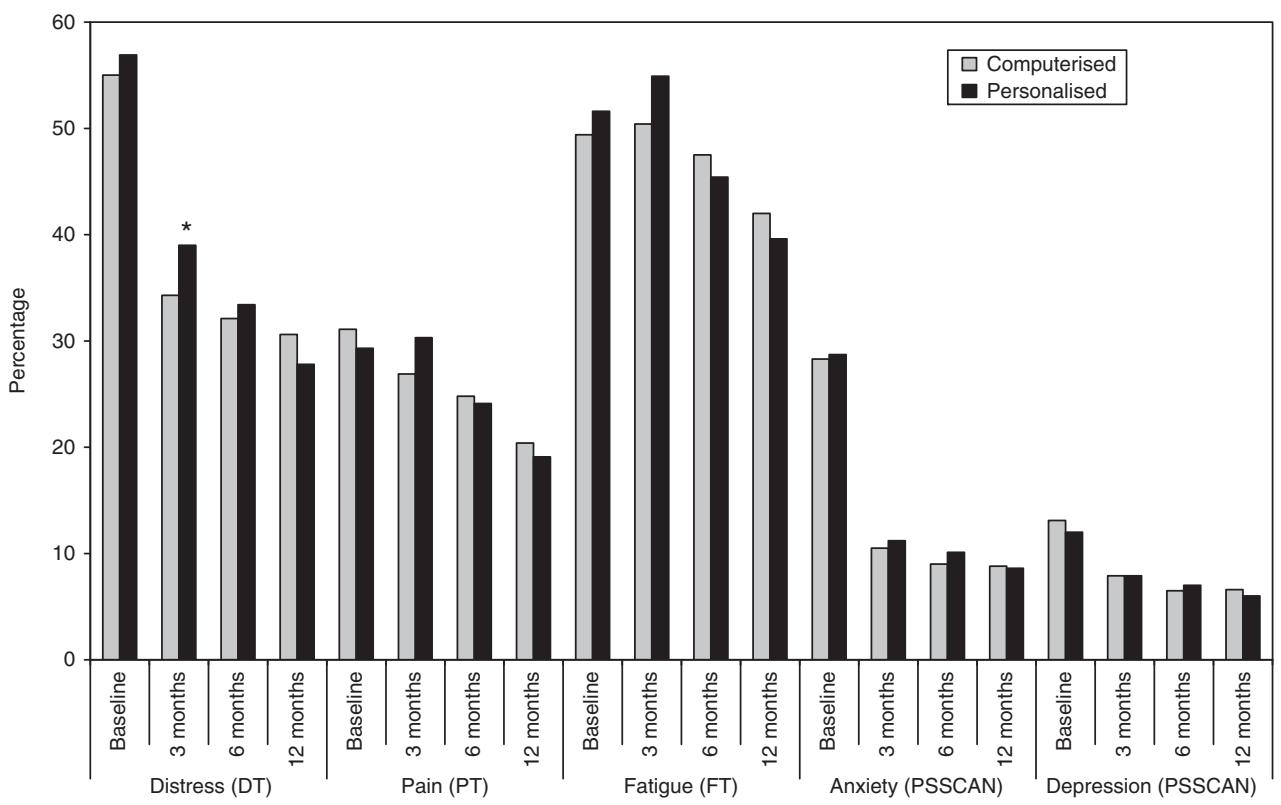

Figure 3 Prevalence of patients reporting scores above the cutoff for each outcome at each time point by triage group.

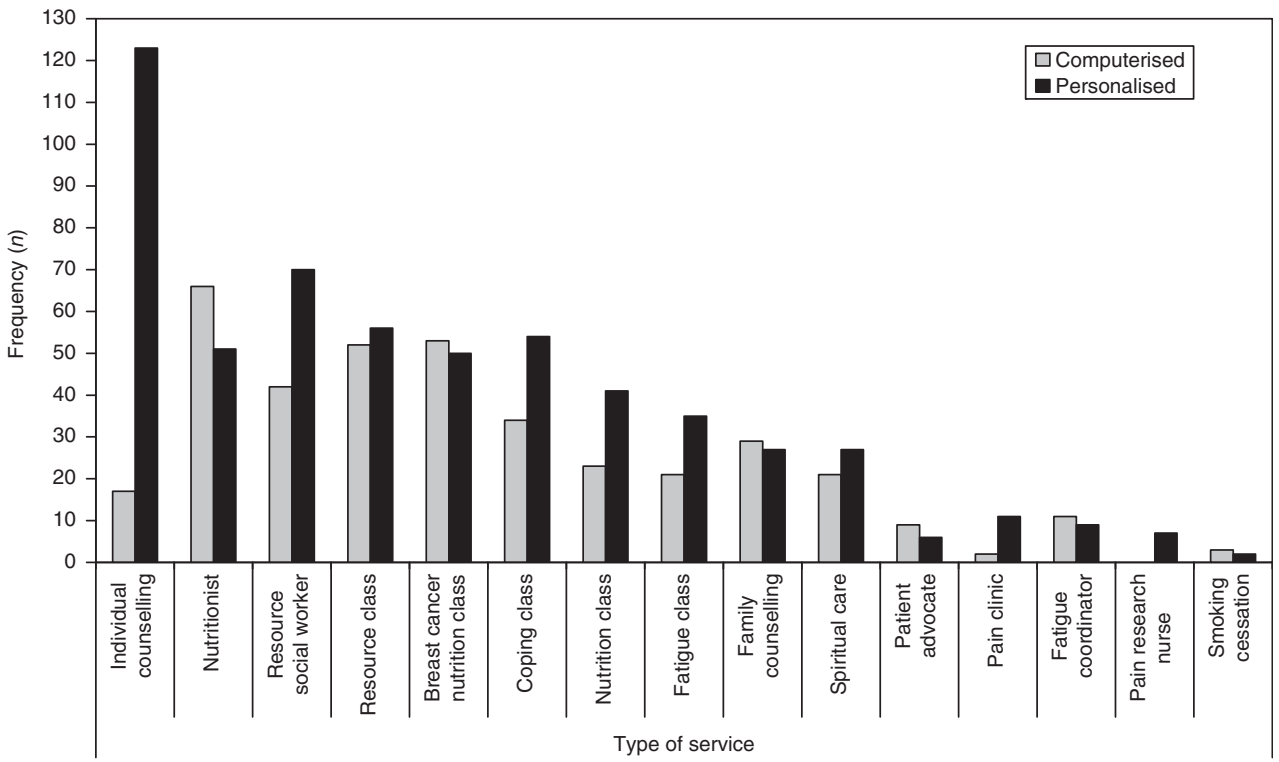

Figure 4 Number of participants accessing each service at least once over 12 months by triage group.

likely to use the services available to them (1213 services vs 825 services accessed). Hence, it was not so much being assigned to either group that was important in improving outcomes, but rather what happened after receiving triage. Those patients who took up referrals improved more over time, no matter which kind of triage they received. This is similar to our previous finding where resource utilisation predicted improvements over time beyond the type of screening received (Carlson et al, 2010). Patients who received personalised triage also accessed a greater number of different services, and used services such as counselling almost nine times as much as those in computerised triage - a huge difference. The increased number of patients accepting referrals in the personalised triage group may be due, in part, to overcoming stigma about counselling or psychological referrals through a oneon-one interaction.

The implication of this finding, that uptake of referrals is the most important determiner of outcomes, suggests that we need to investigate ways to improve uptake of resources, rather than simply focusing on screening alone. We also found that people reporting high anxiety, depression and distress scores were more likely to access services than people reporting low scores, regardless of group assignment. The highest levels of uptake, by far, were by highly depressed and anxious patients in the personalised group.

Taken together, these results suggest that presenting a summary of results to the patient and health-care team followed by a personalised phone call to discuss referral options can prompt patients to access the services offered to them, thus resulting in decreased symptom burden. Keeping in mind that overall group comparisons were not different (and hence personalised triage is not better across all patients than computerised methods), the most likely efficacious and efficient model would be to contact patients high on anxiety and depression to offer personalised triage, and especially women in these categories, allowing those 
Table 3 The proportion of patients in each group reporting that they accessed at least one service at each follow-up time point comparing patients reporting above and below the clinical cutoff on outcomes of interest

\begin{tabular}{|c|c|c|c|c|c|c|c|c|}
\hline \multirow[b]{2}{*}{ Time point } & \multicolumn{4}{|c|}{ Computerised } & \multicolumn{4}{|c|}{ Personalised } \\
\hline & Low & High & $\chi^{2}$ & $P$ & Low & High & $\chi^{2}$ & $P$ \\
\hline \multicolumn{9}{|c|}{ Baseline - 3 months } \\
\hline Distress & 8.2 & 15.9 & 15.07 & 0.000 & 12.6 & 20.2 & 11.29 & 0.001 \\
\hline Anxiety & 11.2 & 15.3 & 3.31 & 0.07 & 13.8 & 24.3 & 18.08 & 0.000 \\
\hline Depre & 11.2 & 20.3 & 8.98 & 0.03 & 15.9 & 4.6 & 5.83 & 0.016 \\
\hline Fatigue & 11 & 1 & 2.25 & 0.134 & 14.2 & 19 & 5.78 & 0.016 \\
\hline Pair & 12.5 & 11.8 & 0.90 & 0.764 & 16.6 & 17.4 & 0.098 & 0.754 \\
\hline \multicolumn{9}{|l|}{$3-6$ months } \\
\hline Distress & 13.8 & 19.3 & 5.56 & 0.018 & 17.9 & 23.2 & 4.81 & 0.028 \\
\hline Anx & I. & 21 & 3.73 & 0.05 & 17 & 36.5 & 24.30 & 0.000 \\
\hline Depression & 14.8 & 26.7 & 8.60 & 0.003 & 18.9 & 32.6 & 9.63 & 0.002 \\
\hline Fatigue & 1.41 & 17.2 & 1.95 & 0.163 & 18.7 & 21.0 & 0.924 & 0.336 \\
\hline Pain & 15.3 & 16.7 & 0.316 & 0.574 & 20.4 & 19.1 & 0.249 & 0.617 \\
\hline \multicolumn{9}{|l|}{$6-12$ months } \\
\hline Distress & 18.5 & 18 & 006 & 0.936 & 23.1 & 22 & 0.018 & 0.894 \\
\hline Anxiety & 18 & 22.9 & 1.192 & 0.275 & 22.2 & 29.6 & 2.744 & 0.098 \\
\hline Depression & 18.4 & 19.7 & 0.064 & 0.800 & 21.3 & 43.5 & 17.77 & 0.000 \\
\hline Fatigue & 17.7 & 19.3 & 0.374 & 0.541 & 22.1 & 24 & 0.498 & 0.480 \\
\hline Pain & 18.6 & 18.0 & 0.045 & 0.832 & 22.9 & 23.1 & 0.006 & 0.940 \\
\hline
\end{tabular}

with lower symptom burden to contact resources suggested through computerised triage themselves.

While this study has several distinct strengths, including the large sample size and long duration of follow-up, there are some limitations. Chiefly, the absence of a randomised usual care comparison group limits the interpretation. However, previous work has already established the feasibility of screening in cancer settings, and the superiority of screening with triage to screening without triage (Carlson et al, 2010), so it seemed somewhat unethical not to offer some form of triage. Another limitation was that triage to services was only provided at the baseline visit. Perhaps providing triage after each follow-up may have resulted in a widening differential effect of the two methods over time.

We relied on the same single-item measures to assess outcomes as used for the screening. The use of single-item screening instruments is valid for detecting outcomes compared with multidimensional tools (Jean-Pierre et al, 2007; Butt et al, 2008; Mitchell, 2010). These tools are more efficient and less burdensome to patients and health professionals (Jean-Pierre et al, 2007; Butt et al, 2008; Mitchell, 2010), and allowed us to use the same measurement system at baseline and follow-up. The service utilisation data were obtained by patient self-report based on specific prompts regarding the usage of each of services. Studies comparing service utilisation obtained by patients self-report $v s$ medical records have generally found good agreement on the part of study participants (Lubeck and Hubert, 2005); however, others suggest that patients tend to underreport their utilisation of health- care services relative to computerised provider records (Ritter et al, 2001).

While this screening for distress program was well integrated into clinic flow, there were still limitations in the area of staff training. Indeed, with many researchers highlighting the importance of intensive staff training for the successful implementation of screening for distress programs (Fitch, 2009; Absolom et al, 2011; Bidstrup et al, 2011; Bultz et al, 2011; Grassi et al, 2011), it is important to recognise that this program fell short of optimal program design in this regard. While most staff attended inservices on screening for distress, no structured formal training in how to read profiles and respond to scores was provided. Future work should examine the combination of triage with significant staff education.

A final limitation of note is the smaller recruitment rate than in our previous work and the loss of almost half of our sample over the 12 months. We relied on centre receptionists to direct patients to the screening kiosks, whereas previously we had a screening assistant located directly in the reception area to aid this process. Given this integration into existing systems, and considering that about $12 \%$ of patients were missed at reception and another $15 \%$ were not interested, achieving accrual of over two-thirds of all patients is acceptable, and probably a pragmatic target to improve upon. Regarding loss to follow-up, the largest group lost was patients who were deceased (15.4\%), but there was also about $10 \%$ we could not contact after three attempts and another $10 \%$ who chose to withdraw. This does limit the generalisability of our findings across all patients, but by using HLM analysis techniques and assuming the missing data were missing at random, we could include all patients who provided at least one assessment in the analysis, mitigating these concerns to a large extent.

In summary, this research further demonstrates that positive screening results must be followed up by comprehensive assessment and intervention to provide significant benefit for patients (Jacobsen, 2007; Bidstrup et al, 2011; Grassi et al, 2011). The overall picture emerging from our research is that Screening for Distress is a viable and helpful intervention with the potential to decrease subsequent symptom burden up to 12 months after diagnosis, but to the greatest extent when patients in need accept referrals to services. While our previous studies have provided evidence for the feasibility and efficacy of routine screening in optimal, controlled conditions; this research begins to support its efficacy when integrated more fully into the clinical environment.

\section{ACKNOWLEDGEMENTS}

Dr Linda Carlson holds the Enbridge Research Chair in Psychosocial Oncology, co-funded by the Alberta Cancer Foundation and the Canadian Cancer Society Alberta/NWT Division. She is also an Alberta Heritage Foundation for Medical Research Health Scholar. This research was supported by grants from the Research Initiatives Program of the Alberta Cancer Research Institute.

Supplementary Information accompanies the paper on British Journal of Cancer website (http://www.nature.com/bjc)

\section{REFERENCES}

Absolom K, Holch P, Pini S, Hill K, Liu A, Sharpe M (2011) The detection and management of emotional distress in cancer patients: the views of health-care professionals. Psychooncology 20(6): 601-608

Accreditation Canada. Qmentum Program 2009 Standards: Cancer Care and Oncology Services. Version 2; 2008

Akechi T, Okuyama T, Akizuki N, Azuma H, Sagawa R, Furukawa TA, Uchitomi Y (2006) Course of psychological distress and its predictors in advanced non-small cell lung cancer patients. Psychooncology 15: 463-473
Andreu Y, Galdon MJ, Dura E, Martinez P, Perez S, Murgui S (2012) A longitudinal study of psychosocial distress in breast cancer: prevalence and risk factors. Psychol Health 27(1): 72-87

Bidstrup PE, Johansen C, Mitchell AJ (2011) Screening for cancer-related distress: summary of evidence from tools to programmes. Acta Oncol 50: 194-204

Bultz BD, Groff SL, Fitch M, Screening for Distress Toolkit Working Group (2009) Guide to Implementing Screening for Distress, the 6th Vital Sign, 
Part A: Background, Recommendations, and Implementation. Canadian Partnership Against Cancer (CPAC)

Bultz B, Johansen C (2011) Screening for distress, the 6th vital sign: where are we, and where are we going? Psychooncology 20: 569-571

Bultz BD, Groff SL (2009) Screening for distress, the 6th vital sign in oncology: from theory to practice. Oncol Exchange 8: 8

Bultz BD, Groff SL, Fitch M, Claude-Blais M, Howes J, Levy K (2011) Implementing screening for distress, the 6th vital sign: a Canadian strategy for changing practice. Psychooncology 20: 463-469

Butt Z, Wagner LI, Beaumont JL, Paice JA, Peterman AH, Shevrin D, Von Roenn JH, Carro G, Straus JL, Muir JC, Cella D (2008) Use of a singleitem screening tool to detect clinically significant fatigue, pain, distress, and anorexia in ambulatory cancer practice. J Pain Symptom Manage 35: 20-30

Carlson LE, Angen M, Cullum J, Goodey E, Koopmans J, Lamont L, MacRae JH, Martin M, Pelletier G, Robinson J, Simpson JS, Speca M, Tillotson L, Bultz BD (2004) High levels of untreated distress and fatigue in cancer patients. Br J Cancer 90: 2297-2304

Carlson LE, Groff SL, Maciejewski O, Bultz BD (2010) Screening for distress in lung and breast cancer outpatients: a randomized controlled trial. J Clin Oncol 28: 4884-4891

Carlson LE, Waller A, Groff SL, Giese-Davis J, Bultz BD (2011) What goes up doesn't always come down: Patterns of distress, physical and psychosocial morbidity in people with cancer over a one year period. Psychooncology; e-pub ahead of print 4 October 2011; doi:10.1002/pon.2068

Cleeland CS, Ryan KM (1994) Pain assessment: global use of the Brief Pain Inventory. Ann Acad Med Singapore 23: 129-138

Couper JW, Love AW, Duchesne GM, Bloch S, Macvean M, Dunai JV, Scealy M, Costello A, Kissane DW (2010) Predictors of psychosocial distress 12 months after diagnosis with early and advanced prostate cancer. Med J Aust 193: S58-S61

Cunningham AJ (1995) Adjunctive psychosocial therapy for cancer: what we know and what we need to know. Chronic Dis Can 16: S13-S18

Cunningham AJ, Edmonds CV (1996) Group psychological therapy for cancer patients: a point of view, and discussion of the hierarchy of options. Int J Psychiatry Med 26: 51-82

Dworkin RH, Turk DC, Farrar JT, Haythornthwaite JA, Jensen MP, Katz NP, Kerns RD, Stucki G, Allen RR, Bellamy N, Carr DB, Chandler J, Cowan P, Dionne R, Galer BS, Hertz S, Jadad AR, Kramer LD, Manning DC, Martin S, McCormick CG, McDermott MP, McGrath P, Quessy S, Rappaport BA, Robbins W, Robinson JP, Rothman M, Royal MA, Simon L, Stauffer JW, Stein W, Tollett J, Wernicke J, Witter J (2005) Core outcome measures for chronic pain clinical trials: IMMPACT recommendations. Pain 113: 9-19

Fitch M (2009) Screening for distress, the 6th vital sign: a Canadian strategy for influencing the agenda for person-centered care: developing a panCanadian Strategy. Psychooncology 18: 29-30

Fitch M (2011) Screening for distress: a role for oncology nursing. Curr Opin Oncol 23: 331-337

Grassi L, Rossi E, Caruso R, Nanni MG, Pedrazzi S, Sofritti S, Sabato S (2011) Educational intervention in cancer outpatient clinics on routine screening for emotional distress: an observational study. Psychooncology 20: $669-674$

Graves KD, Arnold SM, Love CL, Kirsh KL, Moore PG, Passik SD (2007) Distress screening in a multidisciplinary lung cancer clinic: prevalence and predictors of clinically significant distress. Lung Cancer 55: $215-224$

Hauser K, Walsh D (2008) Visual analogue scales and assessment of quality of life in cancer. J Support Oncol 6: 277-282

Holland JC, Bultz BD, National comprehensive Cancer Network (NCCN) (2007) The NCCN guideline for distress management: a case for making distress the sixth vital sign. J Natl Compr Canc Netw 5: 3-7

Jacobsen PB (2007) Screening for psychological distress in cancer patients: challenges and opportunities. J Clin Oncol 25: 4526-4527
Jacobsen PB, Donovan KA, Trask PC, Fleishman SB, Zabora J, Baker F, Holland JC (2005) Screening for psychologic distress in ambulatory cancer patients. Cancer 103: 1494-1502

Jacobsen PB, Ransom S (2007) Implementation of NCCN distress management guidelines by member institutions. J Natl Compr Canc Netw 5: 99-103

Jean-Pierre P, Figueroa-Moseley CD, Kohli S, Fiscella K, Palesh OG, Morrow GR (2007) Assessment of cancer-related fatigue: implications for clinical diagnosis and treatment. Oncologist 12(Suppl 1): 11-21

Jenkins V, Fallowfield L, Saul J (2001) Information needs of patients with cancer: results from a large study in UK cancer centres. $\mathrm{Br} J$ Cancer 84 : $48-51$

Linden W, Vodermaier AA, McKenzie R, Barroetavena MC, Yi D, Doll R (2009) The Psychosocial Screen for Cancer (PSSCAN): Further validation and normative data. Health Qual Life Outcomes 7: 1-8

Linden W, Yi D, Barroetavena MC, MacKenzie R, Doll R (2005) Development and validation of a psychosocial screening instrument for cancer. Health Qual Life Outcomes 3: 54

Lubeck DP, Hubert HB (2005) Self-report was a viable method for obtaining health care utilization data in community-dwelling seniors. J Clin Epidemiol 58: 286-290

Mitchell AJ (2010) Short screening tools for cancer-related distress: a review and diagnostic validity meta-analysis. J Natl Compr Canc Netw 8: 487-494

Mitchell AJ, Vahabzadeh A, Magruder K (2011) Screening for distress and depression in cancer settings: 10 lessons from 40 years of primary-care research. Psychooncology 20: 572-584

Mitchell AJ (2007) Pooled results from 38 analyses of the accuracy of distress thermometer and other ultra-short methods of detecting cancerrelated mood disorders. J Clin Oncol 25: 4670-4681

Morrow GR, Shelke AR, Roscoe JA, Hickok JT, Mustian K (2005) Management of cancer-related fatigue. Cancer Invest 23: 229-239

National Comprehensive Cancer Network CRF Panel (2005) Practice Guidelines in Oncology - v.2.2005: Cancer-Related Fatigue. http:// www.nccn.org/professionals/physician_gls/PDF/fatigue.pdf

National Comprehensive Cancer Network I (2002) Practice Guidelines in Oncology - v.1.2002: Distress Management

Pirl WF, Muriel A, Hwang V, Kornblith A, Greer J, Donelan K, Greenberg D, Temel J, Schapira L (2007) Screening for psychosocial distress: a national survey of oncologists. J Support Oncol 5: 499-504

Raudenbush SW, Bryk AS (2002) Heirarchical Linear Models: Applications and Data Analysis Methods. Sage: Thousand Oaks

Rebalance Focus Action Group (2005) A position paper: screening key indicators in cancer patients: pain as a 5 th vital sign and emotional distress as a 6th vital sign. Cn Strat Cancer Control Bull 7(suppl): 4

Ritter PL, Stewart AL, Kaymaz H, Sobel DS, Block DA, Lorig KR (2001) Selfreports of health care utilization compared to provider records. J Clin Epidemiol 54: 136-141

Sloan JA, Frost MH, Berzon R, Dueck A, Guyatt G, Moinpour C, Sprangers M, Ferrans C, Cella D, Clinical Significance Consensus Meeting Group (2006) The clinical significance of quality of life assessments in oncology: a summary for clinicians. Support Care Cancer 14: 988-998

Vodermaier A, Linden W (2008) Emotional Distress Screening in Canadian Cancer Care: a survey of utilization, tool choices and practice patterns. Oncol Exchange 7: 37-40

Waller A, Williams AD, Groff SL, Bultz BD, Carlson LE (2011) Screening for distress, the sixth vital sign: examining self-referral in people with cancer over a one-year period. Psychooncology; e-pub ahead of print 2 December 2011; doi:10.1002/pon.2102

Wang XS, Fairclough DL, Liao Z, Komaki R, Chang JY, Mobley GM, Cleeland CS (2006) Longitudinal study of the relationship between chemoradiation therapy for non small-cell lung cancer and patient symptoms. J Clin Oncol 24: 4485-4491

Zabora J, Brintzenhofeszoc K, Curbow B, Hooker C, Piantadosi S (2001) The prevalence of psychological distress by cancer site. Psychooncology 10: $19-28$

This work is published under the standard license to publish agreement. After 12 months the work will become freely available and the license terms will switch to a Creative Commons Attribution-NonCommercial-Share Alike 3.0 Unported License. 